\title{
Rotina de famílias de crianças com paralisia cerebral
}

\section{Families of children with cerebral palsy routine}

\author{
Simone Souza da Costa Silva ${ }^{1}$ \\ Fernando Augusto Ramos Pontes ${ }^{1}$
}

\begin{abstract}
RESUMO
A paralisia cerebral (PC) é ocasionada por uma lesão neurológica que afeta o cérebro em seu desenvolvimento inicial, produzindo consequências, essencialmente, motoras, porém, dependendo da área lesionada, o indivíduo pode apresentar comprometimento cognitivo. Crianças com essa deficiência necessitam de cuidados especiais e exigem das famílias uma reestruturação que possibilite o desenvolvimento adequado desses indivíduos. Tendo isto como base investigou-se a rotina de famílias com crianças diagnosticadas com paralisia cerebral. Foi aplicado o Inventário de Rotina (IR) a três mães de crianças com PC atendidas no Serviço Caminhar, do Hospital Universitário Bettina Ferro de Souza, de Belém do Pará. Os resultados apontaram que mães de crianças com $\mathrm{PC}$ tendem a dispor a maior parte do seu tempo aos cuidados com o filho em detrimento do tempo de dedicação a elas mesmas, incluindo cuidados com sua saúde. Este padrão comportamental é gerado por influência dos níveis de comprometimento motor e de autonomia da criança - quanto maior o nível de comprometimento motor, menor o nível de autonomia e maior é a sobrecarga de cuidado da mãe.
\end{abstract}

Palavras-chave: rotinas; famílias; paralisia cerebral.

\footnotetext{
ABSTRACT

Cerebral palsy (CP) is caused by a neurological injury that affects the brain in its early development, producing essentially motor consequences. However,

DOI: $10.1590 / 0104-4060.44688$

1 Universidade Federal do Pará. Programa de Pós-Graduação em Teoria e Pesquisa do Comportamento. Belém, Pará, Brasil. Av. Bernardo Sayão, s/nº. CEP: 66000-000. E-mails: symonufpa@gmail.com; farp1304@gmail.com
} 
depending on the injured area, the individual may have cognitive impairment. Children with this disability need special care and require families' restructuring that allows the proper development of these individuals. On this basis we investigated the routine of families with children diagnosed with cerebral palsy. A Routine Inventory (RI) was applied to three mothers of children with CP treated at the Serviço Caminhar (Walking Service) of the Bettina Ferro de Souza University Hospital in Belém do Pará. The results showed that mothers of children with cerebral palsy tend to have most of their time to care for their children at the expense of devoting less time to themselves, including their health care. This behavioral pattern is generated by the influence of the motor impairment levels and the child's autonomy the higher the motor impairment level, the lower the level of autonomy and the higher the mother's care overload.

Keywords: routines; families; cerebral palsy.

Nos últimos anos tem crescido o número de pesquisas que investigam as rotinas familiares. Este crescimento se deve a dois aspectos fundamentais: o primeiro, à certeza da importância do contexto familiar para o desenvolvimento das pessoas; e o segundo, se refere à compreensão de que a influência exercida pela família sobre a vida destas pessoas está relacionada com o modo como estruturam suas atividades rotineiramente. Neste sentido, o caminho a ser percorrido pelo sujeito desenvolvente está associado com o conjunto de atividades que ele realiza, assim como com as relações estabelecidas por ele durante a realização das atividades. Daí, as pesquisas sobre rotina estarem focalizando na identificação das atividades e das companhias. (XAVIER et al., 2014, SOUZA et al., 2010).

Em termos gerais as pesquisas sobre rotina compartilham a noção de que as atividades consistem em comportamentos repetitivos e observáveis no dia a dia, envolvendo no mínimo dois membros familiares que mantêm regularidade e previsibilidade na vida familiar. (BOYCE et al., 1983. Os estudos sobre as rotinas familiares têm sido realizados em diferentes contextos, como ribeirinhos (SILVA et al., 2010), famílias atendidas por programas sociais (FREIRE et al., 2013), etc. Além de estudos em contextos típicos de desenvolvimento, é possível identificar pesquisas em outros contextos, como em situações pós-traumáticas (BOEHS; FERNANDES, 2013) e em famílias adotivas. (BRANDÃO; CAVALCANTE, 2015). A despeito do interesse nas rotinas das famílias, nota-se carência de pesquisas que investiguem a rotina de famílias de pessoas com deficiência. As evidências empíricas revelam que as famílias vivem em condições de sobrecarga e estresse em virtude das demandas de cuidado gerado pela presença das limitações de um de seus membros. (BARBOSA; BALIEIRO; PETTENGILL, 2012). 
Em termos gerais, os estudos indicam o nível de sobrecarga e estresse das famílias com deficiência a partir de dados obtidos mediante a aplicação de escalas e entrevistas. (PIMENTA; RODRIGUES; GREGUOL, 2010; ROSSET, 2009). Pouco se sabe sobre a sobrecarga dessas famílias a partir das atividades rotineiras executadas por seus membros. Ora, é evidente que a presença de uma pessoa com deficiência em dado contexto acarreta demandas inexistentes em contextos familiares típicos; no entanto, as pesquisas não revelam o que de fato é realizado nessas famílias, que atividades e com quem. Não se pode deixar de considerar que a resposta a estes questionamentos permitirá apontar as possibilidades de aquisições e, consequentemente, de desenvolvimento da pessoa com deficiência e sua família.

As pesquisas sobre famílias de pessoas com deficiência sugerem que as diferentes características que marcam essas pessoas estabelecem dinâmicas na vida diária peculiares; deste modo, os desafios postos à família por uma criança autista são distintos daqueles estabelecidos por uma criança com síndrome de down ou com paralisia cerebral. (PISULA, 1998).

A criança com PC possui um atraso de desenvolvimento neuropsicomotor, podendo causar comprometimento na área motora, sensorial e/ou cognitiva, implicando em alterações no tônus muscular, na qualidade de movimento, nas percepções e na capacidade de apreender e interpretar os estímulos ambientais. Muitas vezes, as sequelas da PC se agravam pelas dificuldades que as crianças apresentam em explorar o meio e em se comunicar com o mundo externo.

A classificação da PC é realizada mediante análise da qualidade do tônus muscular, padrão da expressão motora e região do comprometimento cerebral. O tipo de alteração do movimento observado está relacionado com a localização da lesão no cérebro e a gravidade das alterações depende da extensão da lesão. $\mathrm{O}$ diagnóstico de $\mathrm{PC}$ usualmente envolve retardo ou atraso no desenvolvimento motor, persistência de reflexos primitivos, presença de reflexos anormais e o fracasso do desenvolvimento dos reflexos protetores. (DANTAS et al., 2010).

A paralisia cerebral se revela de forma diversa, não sendo possível encontrar crianças com performances idênticas. Algumas têm alterações leves, insignificantes, que as tornam atrapalhadas ao andar, falar ou usar as mãos. Outras são gravemente prejudicadas pela incapacidade motora grave, impossibilidade de andar, falar, escrever e, muitas vezes, associadas a outras limitações, como, por exemplo, déficits na área cognitiva com prejuízo na aprendizagem, déficits sensoriais (visão e/ou audição), crises convulsivas, entre outras características comportamentais e clínicas. Muitas se tornam dependentes nas atividades da vida diária e na realização de atividades funcionais da vida prática. Entre estes dois extremos existem os casos mais variados. De acordo com a localização das lesões e as áreas afetadas, as manifestações podem ser diferentes. (ALVES DE 
OLIVEIRA; GAROTTI; DE SÁ, 2008; BRAGA, 1999; SCHWARTZMAN, 1992; PFEIFER, 1994).

Existem várias classificações utilizadas, a mais citada é estruturada de acordo com a parte do corpo afetada ou o tipo de movimento manifestado. (MANCINI et al., 2002; MILLER; BACHRARD, 2006; DUARTE, apud MACEDO, 2008). Esta classificação não leva em conta a etiologia ou a patologia do problema, mas caracteriza o tipo de prejuízo motor presente e sumariza os achados em termos das características motoras e topográficas dos prejuízos e das manifestações clínicas do comprometimento. (GIANNI, 2003; MANCINI, 2004).

A descrição topográfica se refere à extensão física das sequelas, podendo ser de três formas: tetraparesia, diparesia e hemiparesia. A tetraparesia é considerada a forma mais grave de $\mathrm{PC}$, em que os membros inferiores e os superiores são afetados de forma assimétrica. Estas características podem ser observadas nos primeiros meses de vida, sendo acentuadas progressivamente à medida que a criança vai se desenvolvendo. (BALADI; CASTRO; MORAIS FILHO, 2007).

A diparesia é caracterizada pelo comprometimento acentuado dos membros inferiores, em detrimento dos membros superiores, ocasionado pela espasticidade da musculatura extensora e adutora das pernas. Comumente observa-se a deficiência em torno do segundo semestre, quando a criança passa a utilizar bem os membros superiores, mas não consegue andar. (GIANINI, 2003; MANCINI, 2004).

A hemiparesia é um tipo de PC que se evidencia em torno dos 4-5 meses, quando a criança começa a manipular objetos. Nestes casos há acometimento de apenas um hemicorpo e, frequentemente, há presença de pés em padrão equinovaro. (BALADI; CASTRO; MORAIS FILHO, 2007).

Os distúrbios de postura e movimento podem ser definidos, segundo Herrero e Monteiro (2008), como a inabilidade do corpo em enfrentar com eficiência os efeitos da gravidade. Isso ocorre devido às dificuldades em manter uma postura e realizar um movimento em razão das alterações de tônus e da presença de padrões anormais de movimento. O que implica em perda de funcionalidade, dependência na maioria de suas atividades do cotidiano e necessidade de sua inclusão em programas de reabilitação contínuos. (SCHWARTZMAN, 1992).

Segundo Ribeiro, Porto e Vandenberghe (2013), mães de crianças com paralisia cerebral estão mais expostas aos efeitos da presença de uma criança com paralisia cerebral do que os pais, provavelmente porque as mães estão mais envolvidas na dinâmica do cotidiano de cuidado do filho do que os pais. Neste sentido, o objetivo do presente trabalho é descrever a rotina de mães de crianças com paralisia cerebral (PC) que utilizam os serviços de atendimento do Serviço de Crescimento e Atendimento Caminhar (Serviço Caminhar), do 
Hospital Universitário Bettina Ferro de Souza (HUBFS), da Universidade Federal do Pará (UFPA), na cidade de Belém.

\section{Método}

\section{Delineamento}

Para realização deste estudo utilizou-se uma metodologia exploratório-descritiva de cunho quantitativo e qualitativo.

\section{Participantes}

Participaram deste estudo três mães de crianças com o diagnóstico de paralisia cerebral com idades de 2, 5, 8 anos e com GMFCS (Gross Motor Function System) de níveis V, II e III, respectivamente. Na Tabela 1 é possível observar os dados sociodemográficos das mães e das crianças com PC. Vale ressaltar que os nomes das participantes aqui apresentados são fictícios com vistas a garantir o anonimato de sua identidade.

TABELA 1 - CARACTERIZAÇÃO SOCIODEMOGRÁFICA DAS MÃES PARTICIPANTES E DE SEUS RESPECTIVOS FILHOS COM PC

\begin{tabular}{|c|c|c|c|c|c|c|c|c|}
\hline \multicolumn{5}{|c|}{ Mães } & \multicolumn{4}{|c|}{ Crianças } \\
\hline Nome & Idade & $\begin{array}{c}\text { Situação } \\
\text { Civil }\end{array}$ & Escolaridade & Ocupação & Sexo & Idade & Peso & GMFCS \\
\hline Maria & 30 & Casada & $\begin{array}{l}\text { Ensino Médio } \\
\text { completo }\end{array}$ & Do lar & $\mathrm{F}$ & 8 & 25 & Nível III \\
\hline Antônia & 25 & $\begin{array}{l}\text { União } \\
\text { Estável }\end{array}$ & $\begin{array}{c}\text { Ensino Médio } \\
\text { incompleto }\end{array}$ & $\begin{array}{c}\text { Estudante/ } \\
\text { do lar }\end{array}$ & M & 5 & 15 & Nível II \\
\hline Ana & 21 & Solteira & $\begin{array}{l}\text { Ensino Médio } \\
\text { completo }\end{array}$ & Do lar & M & 2 & 11 & Nível V \\
\hline
\end{tabular}

FONTE: Os autores (2015).

As mães tinham em média 25,33 ( $\mathrm{DP}=4,5)$ anos. Todas residiam com o pai das crianças, seja por estarem casadas, em união estável ou divorciadas ainda residindo com o ex-marido e pai da criança. Quanto à escolaridade, duas tinham Ensino Médio completo e outra cursava o Ensino Médio, e tinham em comum que suas ocupações eram cuidar do lar e dos filhos. Quanto à renda familiar, todas declararam rendimento mensal de mais de um a dois salários mínimos. Duas famílias foram classificadas como famílias extensas, pois havia 
outros membros além do casal e filhos morando na mesma casa. Nesses casos, residiam além do núcleo familiar avós paternos e tio ou tia paterno. Uma família foi classificada nuclear, casal com filhos.

\section{Ambiente}

A coleta de dados ocorreu nas dependências do Serviço Caminhar, na sala de espera e nos consultórios de fisioterapia e terapia ocupacional.

\section{Instrumentos}

INVENTÁRIO DE ROTINAS (IR): Trata-se da segunda versão de um instrumento construído por Silva et al. (2010), cujo objetivo é obter uma descrição detalhada de um dia típico da semana do participante. O instrumento foi adaptado pelo Laboratório de Ecologia do Desenvolvimento (LED), no formato de uma planilha, na qual constam nas linhas a dimensão tempo, com os períodos de um dia: madrugada, manhã, tarde e noite distribuídos por hora, dispostas em um quadrante composto por quatro quadrantes menores que representavam 15 minutos da hora referida, preenchidos pelo aplicador com a sigla da companhia. Enquanto que nas colunas constam as dimensões de atividade realizada - subdividida em categorias menores geradas a priori -, local, companhia e observações complementares. Em sua aplicação, solicitou-se à entrevistada que descrevesse a sequência de atividades desenvolvidas no dia anterior à aplicação, a companhia (com quem realizou) e o local onde foram realizadas. A análise se deu a partir de categorias definidas previamente à coleta dos dados.

\section{Procedimento}

O projeto de pesquisa foi aprovado pelo Comitê de Ética da Universidade Federal do Pará, com registro 473.140. Após o aceite formal da instituição, as participantes foram selecionadas a partir do diagnóstico de paralisia cerebral do filho, na faixa etária de 0 a 12 anos, atendidos no Serviço Caminhar.

As mães selecionadas foram convidadas a participar de forma voluntária da pesquisa. Antes da aplicação do protocolo, a participante foi informada sobre os objetivos do estudo, a preservação de sua identidade, a necessidade de se gravar o áudio da entrevista, bem como da importância de sua participação. Na sequência foi solicitada sua assinatura no Termo de Consentimento Livre e Esclarecido (TCLE). A aplicação do instrumento se deu em encontro previamente agendado com a mãe enquanto aguardava seu filho que se encontrava em atendimento na instituição. 
O protocolo da coleta seguia com a solicitação à mãe para que descrevesse sua rotina de hora a hora a partir do momento em que despertou no dia anterior. Foram realizadas perguntas a ela como: "Que horas você acordou?", "O que você fez em seguida?", "A que horas você iniciou essa atividade?", "Quanto tempo você permaneceu nessa atividade?", "Quem estava em sua companhia neste momento?", "Em que local essa atividade foi realizada?".

Os dados coletados em áudio foram transcritos e reunidos em documento do Microsoft Office Word ${ }^{\circledR}$. Posteriormente, foram tratados no Microsoft Office Excel ${ }^{\circledR}$ com vistas a organizar o orçamento de tempo das entrevistadas aqui apresentado.

\section{Resultados}

A análise de dados da dimensão Atividade, empreendida pela mãe, gerou as seguintes categorias: Descanso (D), Higiene pessoal (H), Higiene da criança (HC), Alimentação (A), Alimentação da criança (AC), Deslocamento para a criança (DC), Deslocamento pessoal (DP), Tarefas domésticas (TD), Atendimento formal da criança (AFD), Entretenimento (E) e Eventos sociais (ES).

A categoria Descanso referiu-se tanto ao momento de repouso e ócio, quanto ao sono diurno - o sono noturno não foi considerado nesta análise. A categoria Higiene pessoal disse respeito à higiene de benefício próprio, como banho, realização de necessidades fisiológicas e vestir-se. Já a Higiene da criança refere-se a ações de higiene dirigidas às crianças. A categoria Alimentação agrupou as atividades de preparo e realização de café da manhã, almoço, jantar e lanche. Enquanto que a Alimentação da criança foram as referidas atividades empreendidas em prol da criança. As categorias Deslocamento pessoal e Deslocamento para a criança contemplaram as atividades de deslocamento com ou sem o uso de meios de transporte, com objetivos pessoais ou relacionados à criança, respectivamente. A categoria Tarefas Domésticas englobou as atividades da rotina diária de cuidado e arrumação da casa, preparar e servir as refeições diárias para si e aos outros membros da família, além do cuidado ao filho. A categoria Atendimento formal da criança referiu-se à espera e realização do atendimento da criança em centros especializados. Já a categoria Entretenimento compreendeu atividades como assistir TV e/ou DVD, usar computador com fins de lazer e momento de interação com a família. Por fim, a categoria Eventos sociais abarcou os momentos de dedicação ao planejamento, organização e realização de reunião social em casa por ocasião do aniversário da criança. 
O orçamento do tempo dispensado a cada categoria foi definido a partir da soma dos minutos em que a mãe dedicava-se às atividades que a constituem. Este obedecia ao total de minutos entre o momento do despertar matinal e o sono noturno de cada mãe. Posteriormente, foram calculadas as porcentagens correspondentes a cada categoria, obedecendo ao total de $100 \%$ (cem por cento).

A análise da dimensão Companhia gerou a seguinte classificação: Sozinha (S), Criança com PC (C), Filhos (FS), Esposo (E), Família nuclear (FN), Família (F) e Parentes (PR). A categoria Filhos disse respeito à companhia conjunta da criança com PC e outro filho com desenvolvimento típico. A Família Nuclear compreende a companhia do esposo e do filho. Enquanto que a categoria Família engloba tanto a família nuclear quanto a família estendida. Já a categoria Parentes referiu-se à companhia de membros isolados da família estendida. Esta dimensão foi igualmente tratada com o orçamento de tempo a partir do cálculo da porcentagem dos minutos em que as mães passavam junto aos membros de cada categoria.

A dimensão Local contemplou os ambientes em que as atividades foram realizadas e também obedeceu ao cálculo de orçamento de tempo dos minutos em que a mãe permaneceu em determinado local e o posterior cálculo de porcentagem de cada local.

A seguir são apresentados os resultados relativos às dimensões Atividade, Companhia e Local e suas respectivas categorias:

\section{Atividade}

A Figura 1 apresenta o tempo dispensado pela mãe às atividades dispostas nas categorias Descanso, Higiene pessoal, Higiene da criança, Alimentação, Alimentação da criança, Deslocamento para a criança, Deslocamento pessoal, Tarefas domésticas, Atendimento formal da criança, Entretenimento e Eventos sociais, em um dia qualquer da semana, especificamente o anterior ao dia da coleta de dados, podendo ou não ser um dia típico da família.

A categoria Entretenimento foi a mais frequente e representou 66,66\% do tempo na rotina de Antônia, seguida em maior porcentagem por Tarefas domésticas realizadas por Maria com 60,81\% e Descanso de Ana com 34,21\%. A categoria Entretenimento teve este destaque na rotina de Antônia devido ao fato de o dia alvo da coleta ter sido o aniversário da criança, assim, a mãe destinou boa parte do seu dia às atividades relacionadas ao planejamento, organização e realização da festa. Enquanto que a categoria Tarefas domésticas teve esta representatividade na rotina de Maria em decorrência de esta estar em período avançado da gestação de seu segundo filho, assim, ela conta com uma rede de 
FIGURA 1 - TEMPO DISPENSADO ÀS ATIVIDADES REALIZADAS EM UM DIA DA SEMANA

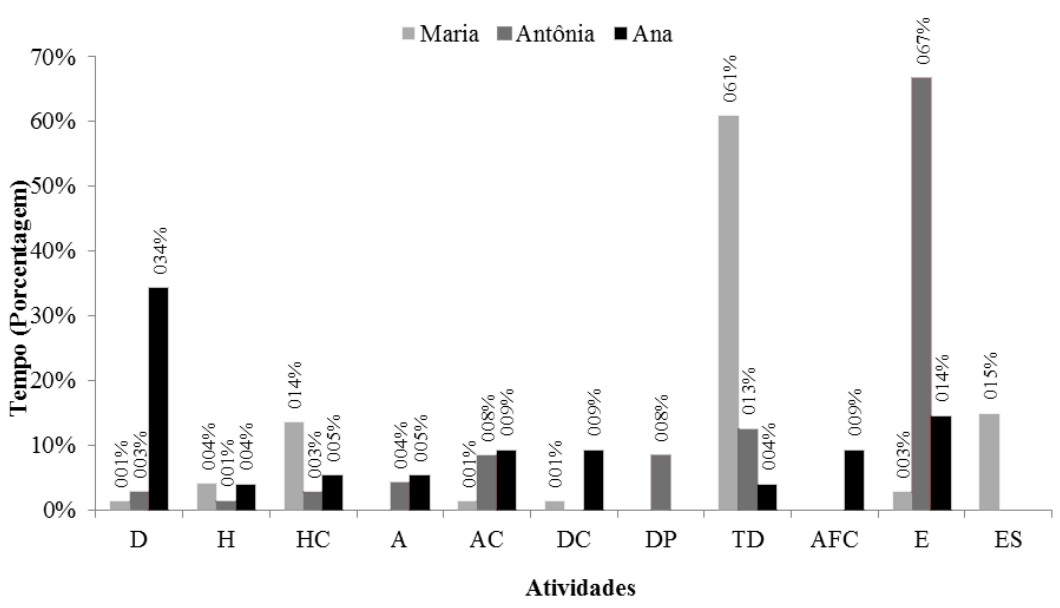

FONTE: Os autores (2015).

suporte familiar que a permite se ausentar de suas habituais atividades rotineiras de cuidado ao filho com PC e dedicação ao lar.

As atividades de Higiene e Alimentação destinadas ao cuidado com as crianças com PC constituem a maior parte da rotina das mães em comparação com as mesmas atividades realizadas em benefício próprio $(\mathrm{H}: 4,05 \%, 1,38 \%$, 3,94\%; HC: $13,51 \%, 2,77 \%, 5,26 \%$; A: 0\%, 4,16\%, 5,26\%; AC: 1,35\%, 8,33\%, $9,21 \%$ ). Isso evidencia a dedicação da mãe em relação aos cuidados básicos com o filho com PC em detrimento dos cuidados pessoais.

Mesmo as atividades que podem ser desprovidas de cuidados aos filhos, como Descanso, Tarefas domésticas, Entretenimento e Eventos sociais, possuem em grande parte a característica de dedicação à criança, seja por meio da companhia ou pelo objetivo de sua realização. Sua frequência se dá do seguinte modo: Descanso (1,35\%, 2,77\%, 34,21\%), Tarefas domésticas, $(60,81 \%, 12,5 \%$, $3,94 \%)$, Entretenimento $(2,7 \%, 66,66 \%, 14,47 \%)$ e Eventos sociais $(14,86 \%$ - Maria).

Outras atividades destinadas exclusivamente às crianças com PC foram Deslocamento para a criança (1,35\% - Maria; 9,21\% - Ana) e Atendimento formal da criança $(9,21 \%$ - Ana). Enquanto Deslocamento pessoal, atividade destinada exclusivamente à mãe, obteve $8,33 \%$ (Antônia).

As ausências de atividades identificadas foram Alimentação de Maria e Atendimento formal da criança de Antônia e Ana. As justificativas são as 
seguintes: Maria relatou que não dispensou momentos específicos à realização de refeições, mas elas foram realizadas concomitantemente a outras atividades, em especial as de Tarefas domésticas; já Antônia e Ana relataram que nos dias alvos da coleta de rotina, as crianças não possuíam atendimentos agendados, pois estes são realizados nos outros dias da semana.

\section{Companhia}

A Figura 2 apresenta as porcentagens de tempo relativas às companhias que a mãe teve enquanto empreendia as atividades constituintes de sua rotina, num dia de semana.

FIGURA 2 - TEMPO DE UM DIA DA SEMANA A PARTIR DAS COMPANHIAS

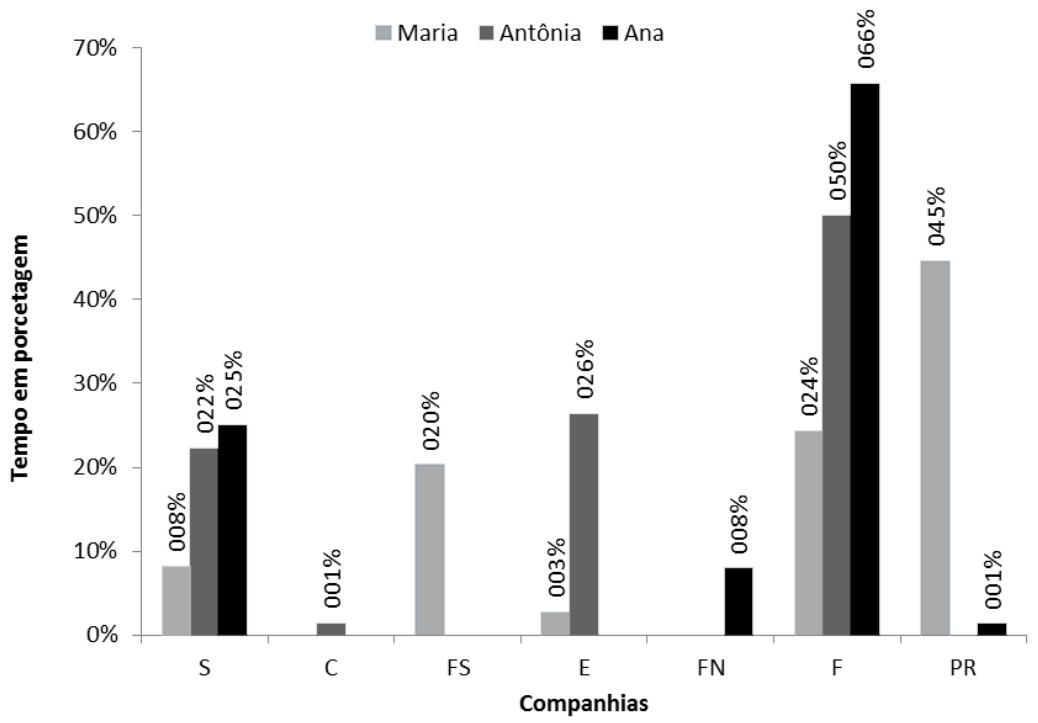

FONTE: Os autores (2015).

As maiores porcentagens nesta dimensão pertencem à Família, com $65,78 \%$ de Ana e 50\% de Antônia, seguida por Parentes, com 44,59\% de Maria. Outros destaques são as categorias de Companhia Esposo com 26,38 (\%) - (Antônia), Família com 24,32\% (Maria), Sozinha com 22,22\% (Antônia) e $25 \%$ (Ana) e Filhos com 20,27\% (Maria).

Em relação às principais companhias das mães, sabe-se que as categorias Família (24,32\%) e Parentes (44,59\%) de Maria foram estritamente relacionadas 
com as atividades de Tarefas domésticas e Eventos sociais, ambos relacionados, por sua vez, com a festa de aniversário da criança com $\mathrm{PC}$ realizada na residência. Enquanto Família (50\%), Esposo (26,38\%) e Sozinha (22,22\%) de Antônia foram em grande parte as companhias na realização da Tarefa doméstica. Já Família $(65,78 \%)$ e Sozinha $(25 \%)$ de Ana foram realizadas em combinações com diversas atividades constituintes da rotina que de fato acontece ao longo dos dias.

Evidencia-se ainda que a Criança como companhia - apresentada apenas por Antônia (1,38 \%) - está presente nas outras categorias de companhia como Filhos, Família Nuclear e Família.

Local

A dimensão Local foi constituída em maioria absoluta pela Residência. Outros locais identificados foram Rua e Centro de atendimento especializado. A rotina de Maria foi constituída pelos locais Residência (98,64\%) e Rua (1,35\%), de Antônia, por Residência (91,64\%) e Rua $(8,33 \%)$, já Ana, por Residência $(90,79 \%)$ e Centro de atendimento especializado (9,21\%).

Estes dados nos permitem observar que mães de crianças com PC tendem a dispor a maior parte do seu tempo aos cuidados com o filho em detrimento do tempo de dedicação a si mesma, incluindo cuidados com sua saúde. Observou-se que a rotina das mães está associada com os níveis de comprometimento motor, logo, do nível de autonomia da criança; quanto maior o nível de comprometimento motor, menor o nível de autonomia e maior é a sobrecarga de cuidado da mãe.

\section{Conclusão}

Um fator identificado como gerador de diferentes padrões comportamentais no cotidiano familiar foi a diferença nos níveis de comprometimentos motor. Este está estritamente relacionado com as habilidades do repertório funcional, bem como com o desempenho da criança em atividades de vida diária. Deste modo, quanto menor o nível de comprometimento motor (níveis 1 e 2 - GMFCS), menor a necessidade da ajuda oferecida pela mãe cuidadora nas tarefas diárias. (MONTEIRO et al., 2012).

Outro aspecto marcante na rotina das mães estudadas foi a presença de fortes redes de suporte social e familiar. Tal aspecto foi responsável por oferecer à mãe uma diminuição na sua sobrecarga de cuidados destinados à criança com PC. Este é um importante fator protetivo na saúde de cuidadoras de crianças 
com paralisia cerebral, o que corrobora com os dados do estudo de Costa et al. (2013), ao apontarem que redes de suporte social contribuem positivamente para a saúde de cuidadoras na medida em que minimizam a sobrecarga do cuidado e contribuem na dinâmica da rotina familiar.

Com o exposto observa-se a importância de intervenções na saúde de mães de crianças com paralisia cerebral com a geração de redes de suporte social, no intuito de diluir a sobrecarga de cuidado que elas enfrentam e oferecer, assim, meios para que possam dedicar tempo à sua saúde, seja no cuidado formal ou informal, como o lazer. Este possibilita que o contato com o filho seja mais que o cuidado diário, e sim reforçador dos vínculos afetivos entre os membros da família.

\section{Considerações finais}

O conhecimento acerca das rotinas de mães de crianças com deficiência, em particular, de mães de pessoas com paralisia cerebral, pode ajudar na medida em que fornece informações que apontam de modo objetivo o grau de sobrecarga vivido pelas cuidadoras. O presente estudo contribui com a literatura uma vez que revela aspectos da vida dessas mulheres que não seriam revelados através de outros instrumentos como escalas e entrevistas. $\mathrm{O}$ dado revela o quanto a vida dessas mães é marcada pelas demandas de seus filhos, que funcionam como elementos estruturantes de seu cotidiano. De fato, mães de crianças com paralisia cerebral organizam sua rotina a partir das necessidades de seus filhos. Neste sentido, atividades básicas como higiene e alimentação se destacam em termos de quantidade de tempo utilizado na sua execução.

A despeito da evidente contribuição deste estudo, observa-se que este apresenta algumas limitações, como número de participantes e ausência de uma análise estatística refinada. Neste sentido, sugere-se que futuras pesquisas sobre o tema envolvam um número maior de participantes, que adotem uma estatística mais sofisticada e que combinem a coleta e a análise de dados quantitativos com a coleta e a análise de informações de natureza qualitativa. Acredita-se que a combinação de dados de fontes diferentes pode oferecer à comunidade científica um panorama mais amplo e consistente sobre o tema. 


\section{REFERÊNCIAS}

ALVES DE OLIVEIRA, A. I.; GAROTTI, M. F.; DE SÁ, N. M. Tecnologia de ensino e tecnologia assistiva no ensino de crianças com paralisia cerebral. Ciências \& Cognição, Rio de Janeiro, v. 13, n. 3, p. 243-262, 2008.

BALADI, A. B.; CASTRO, N. M.; MORAIS FILHO, M. C. Paralisia cerebral. In: FERNANDES, A. C. (Org.). AACD Medicina e Reabilitação: princípios e prática. São Paulo: Artes Médicas, 2007. p. 15-34.

BARBOSA, A. M.; BALIEIRO, M. M. F.; PETTENGIL, M. A. M. Cuidado centrado na família no contexto da criança com deficiência e sua família: uma análise reflexiva. Texto Contexto Enferm, Florianópolis, v. 21, n. 1, p. 194-9, jan./mar. 2012.

BOEHS, A. E.; FERNANDES, G. C. M. Mudanças das rotinas familiares na transição inesperada por desastre natural. Escola Anna Nery (impr), Rio de Janeiro, v. 17, n. 1, jan./mar. 2013.

BOYCE, W. T.; JENSEN, E. W.; JAMES, S. A.; PEACOCK, J. L. The family routines inventory: Theoretical origins. Social Science and Medicine, v. 17, n. 4, p. 201-211, 1983.

BRAGA, L. W. Cognição e paralisia cerebral. Rio de Janeiro: Sarah Letras, 1999.

BRANDÃO, F. H. O.; CAVALCANTE, L. I. C. Rotinas Familiares de Crianças com Necessidades Especiais em Família Adotiva. Teoria e Pesquisa, Brasília, v. 29, n. 3, p. 159-166, abr. 2015.

COSTA, E. A.; PINTO, N. M. A.; FUIZA, A. L. C.; PEREIRA, E. T. Paralisia cerebral e cuidado: o que muda na vida de quem cuida? Oikos: Revista Brasileira de Economia Doméstica, Viçosa, v. 24, n. 1, p. 237-265, 2013.

DANTAS, M. S. A.; COLlET, N.; MOURA, F. M.; TORQUATO, I. M. B. Impacto do diagnóstico de paralisia cerebral para a família. Texto \& Contexto de enfermagem, Florianópolis, v. 19, n. 2, p. 229-237, abr./jun. 2010.

FREIRE, V. R. B. P.; SILVA, S. S. C.; PONTES, F. A. R.; BORGES, J. A. R.; MOURA, M. L. S. Atividades Acadêmicas na Rotina de Crianças Ribeirinhas Participantes do Programa Bolsa Família. Psicologia: Teoria e Pesquisa, Brasília, v. 29, n. 2, p. 159-166, abr. 2013.

GIANNI, M. A. Paralisia cerebral. In: TEIXEIRA, F. N. E.; SAURON, L. S. B.; OLIVEIRA, M. C. Terapia Ocupacional na Reabilitação Física. São Paulo: Rocca, 2003.

HERRERO, D.; MONTEIRO, C. B M. Verificação das habilidades funcionais e necessidades de auxílio do cuidador em crianças com paralisia cerebral nos primeiros meses de vida. Revista brasileira de crescimento e desenvolvimento humano, v. 18, n. 2, p. 163-169, 2008.

MACEDO, P. C. M. Deficiência Física Congênita Saúde Mental. Rev. SBPH, Rio de Janeiro, v. 11, n. 2, p. 35-36, 2008. 
MANCINI, M. C. Gravidade da paralisia cerebral e desempenho funcional. Revista Brasileira de Fisioterapia, v. 8, n. 3, p. 253-260, 2004.

MANCINI, M. C. et al. Comparação do desempenho de atividades funcionais em crianças com desenvolvimento normal e crianças com paralisia cerebral. Arquivos de Neuropsiquiatria, v. 60, n. 2-B, p. 446-452, 2002.

MILLER, F.; BACHRARD, S. J. Cerebral Palsy: a complete guide for caregiving. 2. ed. Baltimore, Maryland: The John Hopkins University Press, 2006.

MONTEIRO, J. A.; VASCONCELOS, T. B.; SILVA, R. L. M.; CAVALCANTE, L. I. C. et al. Avaliação do nível de independência nas atividades de vida diária da criança com paralisia cerebral: um estudo de caso. Cad. Ter. Ocup. UFSCar, São Paulo, v. 20, n. 1, p. 129-141, 2012.

PFEIFER, L. I. Comprometimento motor e habilidades cognitivas em crianças com sequelas de paralisia cerebral. Dissertação (Mestrado em Educação Especial) - Universidade Federal de São Carlos, São Paulo, 1994.

PIMENTA, R. A.; RODRIGUES, L. A.; GREGUOL, M. Avaliação da Qualidade de Vida e Sobrecarga de Cuidadores de Pessoas com Deficiência Intelectual. Revista Brasileira de Ciências da Saúde, João Pessoa, v. 14, n. 3, p. 69-76, 2010.

PISULA, E. Stress in Mothers of Children with Developmental Disabilities. Polish Psychological Bulletin, v. 29, p. 305-11, 1998.

RIBEIRO, M. F. M.; PORTO, C. C.; VANDENBERGHE, L. Parental stress in families of children with cerebral palsy: an integrative review. Ciência \& Saúde Coletiva, Rio de Janeiro, v. 18, n. 6, p. 1705-1715, 2013.

ROSSET, M. O. S. A sobrecarga na família de crianças com paralisia cerebral. Dissertação (Mestrado) - Universidade de São Paulo, Ribeirão Preto, 2009.

SCHWARTZMAN, J. S. Paralisia cerebral. Temas sobre desenvolvimento. Memnon, São Paulo, v. 6, p. 3-5, 1992.

SILVA, S. S. C.; PONTES, F. A. R.; SANTOS, T. M.; MALUSCHKE, J. B.; MENDES, L. S. A.; SILVA, S. D. B. Rotinas Familiares de Ribeirinhos Amazônicos: Uma Possibilidade de Investigação. Psicologia: Teoria e Pesquisa, Brasília, v. 26, n. 2, p. 341-350, abr. 2010.

SOUZA, M. D.; SOUSA, A. O.; PARENTE, A. C. B. V.; MARTINS, M. C. C. Avaliação da sobrecarga em familiares cuidadores de pacientes esquizofrênicos adultos. Psicologia em estudo, Maringá, v. 15, n. 3, p. 639-647, jul. 2010.

XAVIER, D. M.; GOMES, S. S.; LUNARDI, V. L.; PINTANEL, A. C.; ERDMANN, A. L. A família na Unidade de Pediatria: convivendo com normas e rotinas hospitalares. Revista Brasileira de Enfermagem, Brasília, v. 67, n. 2, p. 181-186, abr. 2014.

Texto recebido em 13 de janeiro de 2016. Texto aprovado em 13 de janeiro de 2016. 